\section{Abrechnungsbarone und andere Wissenschaftler}

\author{
S. Pfaundler
}

\begin{abstract}
Übrigens, kennen Sie die Geschichte vom Baron und seiner goldenen Schaufel? Ich komme später nochmals darauf zurück. Als naturwissenschaftlich Ausgebildeter bin ich nämlich gewohnt, dass wir Daten brauchen, Facts (altdeutsch: Tat-Sachen), um wirksam und zweckmässig arbeiten zu können, nicht Geschichten. Also habe ich von jeher gelernt, Daten zu sammeln. Da Mathematik nie meine Stärke war, wurde ich nicht Ökonom und war ich immer froh, dass es für die mühsame Auswertung meiner Daten ausgebildete Spezialisten gibt. Diese können brillante Statistiken (auch solche, die sogar ich wieder verstehe) erstellen und mir wichtige Rückmeldungen $\mathrm{zu}$ meinen Datensammlungen geben.
\end{abstract}

Das hat sich im Laufe der Zeit gut eingespielt, es ist eine Kultur entstanden, in der ich meine Angaben liefere und die Experten der Zahlenkunst aus diesen vielfältige Ergebnisse entwickeln. Ein Musterbeispiel bewährter Kooperation, wie das ja in etlichen Branchen selbstverständlich ist. Vom anschliessenden Austausch konnten beide Seiten meist viel lernen, und wir wissen in gegenseitigem Respekt um unsere eigenen und gemeinsamen Stärken. So fand interdisziplinäre Wissenschaft statt.

Apropos: Da der eingangs erwähnte Baron einige Ländereien geerbt hatte und diese gewissenhaft pflegen und bewirtschaften wollte, war speziell ein kräftiger, erfahrener Landwirt sein wichtigster Partner. Der Baron schätzte jenen sehr ob seiner Kenntnisse und seines Fleisses. Denn auch er verlangte von sich diese Tugenden. Andererseits hatte er natürlich seine Macken, so wie der fleissige Landwirt bekannt war für seinen Zorn und seine Ungeduld.

$\mathrm{Zu}$ den Facts: Je mehr ich arbeite, um so mehr interessieren mich elementare, grundsätzliche Fragestellungen. Meine Kolleginnen und ich produzieren inzwischen jedoch dermassen viele Daten, dass ich

Korrespondenz:

Dr. med. Sebastian Pfaundler

Fläscherstrasse 37

CH-7310 Bad Ragaz

E-Mail: s.pfaundler@psyqual.ch
Zum elektronischen Datenmanagement in der ärztlichen Praxis

Mit TARMED wird die elektronische Abrechnung Realität und Selbstverständnis (Pflicht). Davon sind auch Behandlungsdaten betroffen.

Aus diesen Daten lassen sich wichtige Informationen für das Gesundheitswesen gewinnen. Darum ist das Interesse an unseren Daten von verschiedenen Seiten auch sehr gross (Markt). Die Datenhoheit liegt immer noch beim Datenerbringer (Arzt).

Aus Gründen der notwendigen Anonymisierung und gezielten Datenverarbeitung werden künftig Clearing-Stellen und Trust-Centers im Datenweg zwischen Dienstleistern und Zahlstellen eingeschaltet sein. Dort werden u.a. Dateneigner und Datenbezieher mittels voneinander verschiedener Codes (Clearing-Nummern) verschlüsselt beziehungsweise identifiziert.

Je nach unserer Beteiligung an derartigen Clearing-Stellen und Trust-Centers werden wir mehr oder weniger beim Prozess der Datenauswertung und -interpretation mitbestimmen können.

selber nicht mehr in der Lage bin, bei der Auswertung dieses Materials mitzureden. Dafür werden heute Clearing-Stellen gegründet, die ihrerseits begierig auf meine Daten warten, damit sie diese verwerten und die Resultate anschliessend mir und anderen Interessierten unterbreiten können. Selbstverständlich kostet das etwas.

Nochmals zurück zum Baron, der eines Tages seine meistgebrauchte, verbeulte und zerkratzte Schaufel vergolden liess. Der tüchtige Landwirt hatte dieses Werkzeug schon des längeren gesucht und kam nun aufgebracht zum Landbesitzer: "Was soll der Blödsinn?", rief er ungehalten und fuchtelte wild mit der glänzenden Schaufel herum. "Das hat seine Gründe, mehr will ich Dir jetzt nicht sagen", antwortete der Baron geheimnisvoll. Der wütende Mann geriet darob noch mehr ins Toben, wobei ihm tragischerweise das funkelnde Werkzeug entglitt und den Baron so unglücklich am Halse traf, dass dieser in die Knie sank und alsbald verblutete.

Wir sind bei den Tatsachen: Trust-Centers sind am Entstehen, die wachsam darauf schauen, dass niemand die Daten des anderen ohne dessen Einwilligung verwenden kann. Dazu müssen diese meine Angaben professionell verschlüsseln. $\mathrm{Zu}$ guter Letzt bin ich sowohl Lieferant als auch Kunde von meinen Datenfluten. Inzwischen tummeln sich hier natürlich auch andere Interessenten mit eigenen Fragestellungen $\mathrm{zu}$ meinen Daten und Resultaten aus meinen Daten. So einfach entstehen Angebot und Nachfrage, neue Marktplätze. 
Wie der Goldschmied später erzählte, hatte der Landbesitzer aus Respekt und Anerkennung für die langjährige Allianz mit seinem fleissigen Partner diesem sein Werkzeug vergolden lassen und beim nächsten festlichen Anlass feierlich übergeben wollen. Leider war es ihm nicht mehr vergönnt, diese Mitteilung rechtzeitig anzubringen.

Betreffend meine Datenlieferungen baue ich immer noch auf partnerschaftliche Kooperation. Ich will mit denen zusammenarbeiten, die meine Anga- ben verwerten und die mir rückmelden können, was die Ergebnisse aus verschiedener Perspektive aussagen. Übrigens bin ich als Arzt (Psychiater und Psychotherapeut) so weit erfolgreich. Ich werde dennoch praktisch nie von meinen Patienten direkt bezahlt. Krankenversicherungen haben dies freundlicherweise landauf landab übernommen. Weil ich wissen will, was diese an meinen Zahlen interessiert, suche ich den Dialog. Vielleicht geht es anderen Datenlieferantinnen ähnlich. 